\title{
Frequency of Bruxism among School Children in Tirana City
}

\author{
Alketa Qafmolla, Ruzhdie Qafmolla \\ Faculty of Stomatology, Tirana, Albania
}

\begin{abstract}
The term bruxism is said to come from the Greek word "brychein," which means "to grind or gnash the opposing rows of upper and lower molar teeth." Per recent classification of sleep disorders, bruxism is defined as a sleep-related movement disorder. This cross-sectional descriptive study was done on 226 4-12 year old children, who were referred to our dental clinic in Tirana city 2014-2015. After acquiring the parents' informed consent, a questioner was administrated to all participants. A total of 226 children were included in the study. Fifty five (60\%) patients were girls and $40(38.2 \%)$ boys with a mean age of patients was 7.4 ( \pm 2.4$)$ years. Bruxism began in these children at the age of $4.5( \pm 2.5)$ years. As reported by parents, 57 (25\%) 95\%CI (19.5-31.2). Parasomnias were stated by parents in $50 \%$ of children, drooling being the most (25\%), and snoring the least (5\%). Effective management of bruxism relies on the recognition of potential causative factors associated with the condition. For example, since daytime or diurnal bruxism may be confounded by factors such as stress, distress, and other psychosocial parameters, considering interventions such as habit modification, relaxation therapy, biofeedback, or counseling may be appropriate.
\end{abstract}

Keywords: children, bruxism, disorder, psychological factors, parasomnia

\section{Introduction}

The term bruxism is said to come from the Greek word "brychein," which means "to grind or gnash the opposing rows of upper and lower molar teeth." Per recent classification of sleep disorders, bruxism is defined as a sleep-related movement disorder. The Glossary of Prosthodontic Terms (GPT-8) defines bruxism as parafunctional tooth grinding habit consisting of involuntary rhythmic or spasmodic nonfunctional gnashing, grinding, or clenching of teeth. $(1,2)$ Behavior that occurs during the day is termed awake or diurnal bruxism and can consist of semivoluntary clenching or lateral tooth grinding movement. Grinding or clinching that occurs at night is termed sleep bruxism. $(3,4)$ Early theories suggesting that bruxism was related to general malocclusion have been disproven, although, in some isolated cases, tooth interferences may play a role in its onset. What has emerged in the last 10 years from extensive research is a broader hypothesis defining the genesis and pathogenesis of bruxism. The factors that have found traction from these studies include genetic predisposition, sleep architecture (eg, micro arousals occurring during sleep), psychological factors such as distress and anxiety, environmental factors, CNS catecholaminergic levels, brain trauma, brain disease, drug effects (stimulatory drugs: ecstasy, caffeine, or serotonin reuptake inhibitors, anxiolytic medication, or dopaminergic drugs, etc), and autonomic nervous system function (pattern generation). Bruxism is also considered a natural behavior that stimulates salivation during sleep. $(5,6)$ One experimental study involving induction of esophageal acidification has shown that bruxism episodes increase with acid stimulation, suggesting that reflux may cause bruxing behavior in individuals with this stomach abnormality.(7)

\section{Material and Methods}

This cross-sectional descriptive study was done on 226 4-12 year old children, who were referred to our dental clinic in Tirana city 2014-2015. After acquiring the parents' informed consent, a questioner was administrated to all participants The questionnaire contained the information about the age, sequence of birth, medical history and also parents' age, education, and job. The second part was questions related to the time of bruxism during the day or night, manifestation of distressing events in the family (such as parental divorce, birth of new baby, start going to school or death of child's loved one), family history of bruxism, temporomandibular joint disorder, parasomnias, oral habits (nail biting, pacifier sucking and lip or thumb sucking) and psychological disorders. Temporomandibular joint disorders were considered as jaw clicking, pain or tenderness in muscles and limitation in mouth opening. Items related to psychological disorders include irritability, seclusion, sadness, neophobia, crying easily, gazing at one point, hyperactivity, obsession, depression, acrophobia or lygophobia and finally parasomnias' items include drooling, sleep talking, sleep walking, mouth breathing and snoring. Chi-square test was used to compare the differences in proportion and Pearson correlation was used to assess the relationship between variables. A p-values $<0.05$ was considered as statistically significant.

\section{Results and Discussion}

A total of 226 children were included in the study. Fifty five $(60 \%)$ patients were girls and $40(38.2 \%)$ boys with a mean age of patients was $7.4( \pm 2.4)$ years. Bruxism began in these children at the age of $4.5( \pm 2.5)$ years. As reported by parents, 57 (25\%) 95\%CI (19.5-31.2) children showed bruxism as a parafunctional habit (fig. 1). There was no significant relation between the gender, the sequence of birth, father's age, and parents' education and father's job with the prevalence of bruxism. $9 \%$ of children had fathers with bruxism history, $8 \%$ mothers with bruxism history and $2 \%$ of children had both parents bruxism history. A significant correlation was found between the family history of bruxism and the prevalence of it $(\mathrm{p}<0.01)$. The prevalence of bruxism was $22 \%$ in children without family history of bruxism and $85 \%$ in children with both parents history of 


\section{International Journal of Science and Research (IJSR) \\ ISSN (Online): 2319-7064 \\ Index Copernicus Value (2013): 6.14 | Impact Factor (2015): 6.391}

bruxism. According to parents' report, $87 \%$ of children with bruxism had a history of distressing events in their life, and $13 \%$ of children with bruxism didn't report any history of distressing events in their life. The difference between these 2 groups was significant $(\mathrm{p}<0.01)$. Parasomnias were stated by parents in $50 \%$ of children, drooling being the most (25\%), and snoring the least (5\%) (fig. 2). Bruxism was seen in $32 \%$ of children with parasomnias and merely in $16 \%$ of subjects without any sleep disorders. There was a significant difference between these 2 groups, and there was a correlation between drooling, sleep walking and bruxism $(\mathrm{p}<0.01)$. Oral habits were reported in $20 \%$ of children. Nail biting was the most frequent habit with a prevalence of $11 \%$. The prevalence of bruxism in children with TMD was $61 \%$ and in children without TMD 20\%; the difference was significant $(\mathrm{p}<0.01)$. No significant relationship was found between the history of asthma and allergy or systemic disease with bruxism. In our study psychological disorders, i.e. irritability (35\%), crying easily $(27 \%)$ and hyperactivity $(17 \%)$ were the most frequent ones. The diagnosis of bruxism is descriptive in nature, contingent on the presence of symptoms such as acknowledged tooth grinding, pain in the TMJ or jaw musculature, temporal headache, tooth hypersensitivity or mobility, and poor sleep quality. These subjective symptoms are coupled with clinical signs such as abnormal tooth wear, tongue indentations, the presence of a linea alba along the biting plane of the buccal (cheek) mucosa, gum recession, masseter hypertrophy, and/or broken fillings or teeth. Attempts have been made to provide a more formal definition of nocturnal bruxism based on sleep architecture and the number of episodes occurring during different phases of sleep (8). However, for some sleep variables their sensitivity and specificity is problematic. Chronic bruxism, based on published reports, can cause mild-to-severe tooth wear, tooth cusp fracture, attached gingival recession, tooth mobility, fractured restorations, masticatory muscle pain, and temporomandibular disorders. Additionally, the literature includes other possible effects such as jaw opening limitation, temporal headache, ear symptoms (eg, plugging, tinnitus, subjective hearing loss), migraines, neck pain, insomnia, and depression. $(9,10)$ In a retrospective analysis of parent report surveys, Insana and colleagues found a high prevalence of sleep-bruxism among preschool children $(36.8 \%)$. In addition, in a subset preschoolers who received additional behavioral and neurocognitive assessments, bruxism was also associated with increased internalizing behaviors and increased health problems, which in turn were associated with decreased neurocognition. (11-13) The researchers concluded that pediatric sleep-bruxism may serve as a warning sign for possible adverse health conditions, and the need for early intervention. $(14,15)$.

\section{Conclusion}

The diagnosis of bruxism is descriptive in nature, contingent on the presence of symptoms such as acknowledged tooth grinding, pain in the TMJ or jaw musculature, temporal headache, tooth hypersensitivity or mobility, and poor sleep quality. These subjective symptoms are coupled with clinical signs such as abnormal tooth wear, tongue indentations, the presence of a linea alba along the biting plane of the buccal (cheek) mucosa, gum recession, masseter hypertrophy, and/or broken fillings or teeth. In diagnosing bruxism, particularly as a sleep disorder, their needs to be a welldefined exclusion criteria that separates bruxism from other sleep disorders such as sleep apnea, epilepsy, and REM disorder behaviors. Effective management of bruxism relies on the recognition of potential causative factors associated with the condition. For example, since daytime or diurnal bruxism may be confounded by factors such as stress, distress, and other psychosocial parameters, considering interventions such as habit modification, relaxation therapy, biofeedback, or counseling may be appropriate. In the patient with sleep bruxism (which does not appear to be impacted by psychological or psychosocial factors), appropriate intervention might include appliance therapy and medication. In patients with medication or drug-induced bruxism, medication withdrawal or a change of medication type to one less likely to cause bruxism should be considered. If street drugs are involved, intervention should include drug and perhaps psychological counseling.

\section{References}

[1] The glossary of prosthodontic terms. The Academy of Prosthodontics. J Prosthet Dent. 1994 Jan. 71(1):41112. (Medline).

[2] (Guideline) Shilpa S, et al. Bruxism: a literature review. J Indian Proshodont Soc. 2010/09. 10(3):141-148.

[3] Thie NM, Kato T, Bader G, Montplaisir JY, Lavigne GJ. The significance of saliva during sleep and the relevance of oromotor movements. Sleep Med Rev. 2002 Jun. 6(3):213-27. (Medline).

[4] Ohmure H, Oikawa K, Kanematsu K, Saito Y, Yamamoto T, Nagahama H. Influence of experimental esophageal acidification on sleep bruxism: a randomized trial. J Dent Res. 2011 May. 90(5):665-71. (Medline).

[5] Lobbezoo F, Lavinge GJ, Tanguay R, Montplaier JY. The effect of the catecholamine precursor L-dopa on sleep bruxism: a controlled clinical trial. Mov Disord. 1997;12:73). Mov Disord. 1997. 12:73.

[6] Bruxism. wikipedia. Available at http://en.wikipedia.org/wiki/Bruxism. Accessed: 11.15.2011.

[7] Lobbezoo F, Soucy JP, Hartman NG, Montplaisir JY, Lavigne GJ. Effects of the D2 receptor agonist bromocriptine on sleep bruxism: report of two singlepatient clinical trials. J Dent Res. 1997 Sep. 76(9):16104. (Medline).

[8] Lobbezoo F, Lavinge GJ, Tanguay R, Montplaier JY. The effect of the catecholamine precursor L-dopa on sleep bruxism: a controlled clinical trial. Mov Disord. 1997. 12:73.

[9] Lobbezoo F, Van Denderan RJ, et al. Reports of SSRIassociated bruxism in the family physician office. $J$ Orofac Pain. 2001. 15:340-346.

[10] Manfredini D, Lobbezoo F. Role of psychosocial factors in the etiology of bruxism. J Orofac Pain. spring/2009. 23(2):

[11]Lavigne GJ, Rompre PH. Lower number of Kcomplexes ande K-alphs in sleep bruxism: a controlled quantitative study. J Orofac Pain. 15(1):64-71. 


\section{International Journal of Science and Research (IJSR) \\ ISSN (Online): 2319-7064 \\ Index Copernicus Value (2013): 6.14 | Impact Factor (2015): 6.391}

[12] Lavigne GJ, Khoury S, Abe S, Yamaguchi T, Raphael $\mathrm{K}$. Bruxism physiology and pathology: an overview for clinicians. J Oral Rehabil. July/2008. 35(7):476-494.

[13] Lavigne GJ, Rompré PH, Montplaisir JY. Sleep bruxism: validity of clinical research diagnostic criteria in a controlled polysomnographic study. $J$ Dent Res. 1996/01. 75(1):546-552.

[14] Insana SP, Gozal D, McNeil DW, Montgomery-Downs HE. Community based study of sleep bruxism during early childhood. Sleep Med. 2013 Feb. 14(2):183-8. (Medline). (Full Text).

[15] Hoz Aizpurua J, Díaz Alonso E, LaTouche Arbizu R, Mesa Jiménez J . Sleep bruxism. Conceptual review and update. Med Oral Patol Oral Cir Bucal. 2011. 16(2):e231-238.

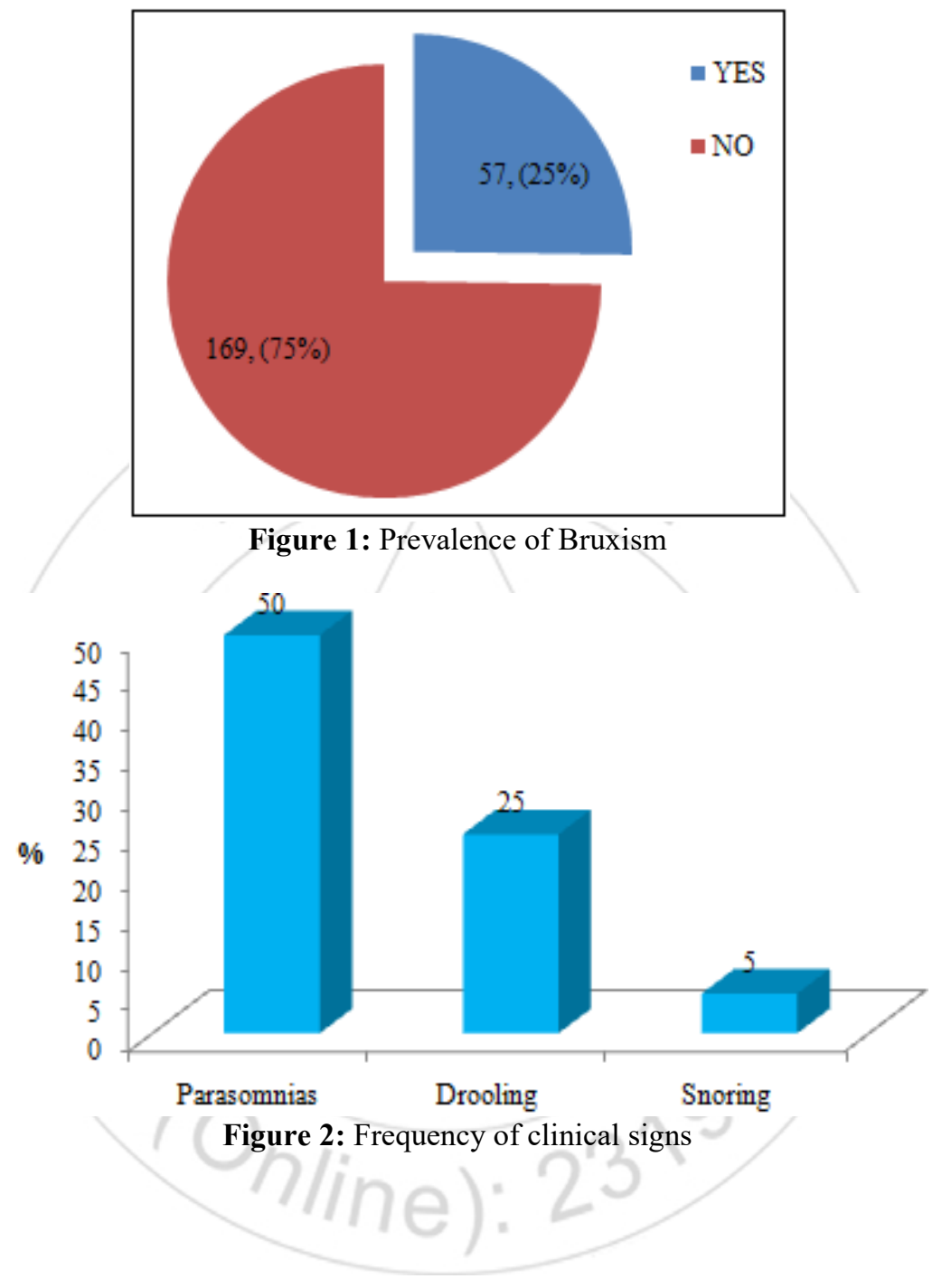

Volume 5 Issue 4, April 2016 